\title{
Effects on the right ventricle, pulmonary vasculature, and carotid bodies of the rat of exposure to, and recovery from, simulated high altitude
}

\author{
D. HEATH, C. EDWARDS, M. WINSON, and P. SMITH \\ The Department of Pathology, University of Liverpool
}

\begin{abstract}
Three groups of 10 adult male Wistar albino rats were studied. The first was kept for five weeks in a hypobaric chamber exposed to a barometric pressure of $380 \mathrm{mmHg}$, equivalent to a simulated altitude of $5,500 \mathrm{~m}$ above sea level. The second was exposed to the same barometric pressure for five weeks and then allowed to recover in room air for a further period of five weeks. The third group acted as controls and was kept at normal barometric pressure throughout. At necropsy right ventricular weight was expressed as an inverse ratio of left ventricular weight (LV/RV ratio). The thickness of the media of the pulmonary trunk was expressed as a ratio of that of the aorta. The volumes of the carotid bodies were measured by applying Simpson's rule to histological sections. In the first group, exposed to chronic hypoxia without relief, there was hypertrophy of the right ventricle and media of the pulmonary trunk and there was an increase of carotid body volume, as compared with the values obtained in the controls. In the recovery group these three measurements had returned almost to normal. The results appear to be applicable to hypoxic cor pulmonale in man. They suggest that hypoxia rapidly produces pulmonary hypertension and its morbid anatomical associations while recovery is equally rapid once the hypoxic stimulus is removed.
\end{abstract}

Chronic hypoxia, irrespective of whether it is brought about by life at high altitude or by respiratory disease, is a potent cause of pulmonary hypertension. The elevated pulmonary arterial pressure is associated with right ventricular hypertrophy and hypoxic hypertensive pulmonary vascular disease (Heath, Brewer, and Hicken, 1968), and the chronic hypoxia also induces enlargement of the carotid bodies (Heath, Edwards, and Harris, 1970 ; Edwards, Heath, and Harris, 1971 a ; Edwards et al., 1971b). In this experiment we set out to determine if these changes were reversible.

\section{MATERIALS AND METHODS}

Three groups of adult male Wistar albino rats were studied, each consisting of 10 animals. The first group was kept under hypoxic conditions in a hypobaric chamber exposed to a barometric pressure of 380 $\mathrm{mmHg}$ for five weeks after which time they were killed (Fig. 1). The animals were exposed to room air daily for some 20 minutes while they were being cleaned and given fresh food and water. These conditions simulated an altitude of $5,500 \mathrm{~m}$ above sea level. These animals were designated the 'hypoxic group'. The second group was kept under identicale hypoxic conditions for five weeks and was ther? allowed to survive for a further five weeks in room. air (Fig. 1). They comprised the 'recovery group' The third group consisted of control animals which

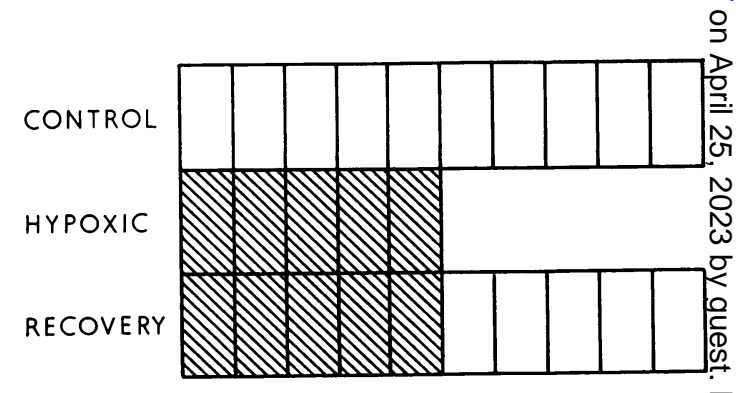

FIG. 1. Diagram of design of experiment. Each ope⿳⺈.9. rectangle represents one week when the animals of tho group breathed room air at normal sea-level barometrif pressure. Each shaded rectangle represents one week when the animals of that group breathed air in the hypobarie chamber at a simulated altitude of 5,500 m (c. 18,000 fo above sea level. 
were kept in room air for the whole 10 -week period of the experiment (Fig. 1). All three groups of animals were allowed free access to food and water. Their initial and final body weights are shown in Table $I$.

At necropsy the thoracic viscera were removed intact from the chest, and the lungs were distended with $4 \%$ formaldehyde until the pleural surface was just smooth. After fixation the free wall of the right cardiac ventricle was dissected off the rest of the heart and weighed separately, according to the method of Fulton, Hutchinson, and Jones (1952). The left ventricle and the interventricular septum were weighed together. The right ventricular weight was expressed as an inverse ratio of the left ventricular weight. This is referred to hereafter as the LV/RV ratio. This gave an indication of any degree of right ventricular hypertrophy present which was independent of total cardiac or body weight.

Rings of pulmonary trunk and aorta were cut just above the levels of the pulmonary and aortic valves respectively. Transverse sections were cut and stained by the Humberstone method for elastic tissue with a van Gieson counterstain to demonstrate smooth muscle and collagen. Ten measurements of the thickness of the media of the pulmonary trunk were made and a mean medial thickness was calculated. A mean medial thickness of the aorta was calculated in the same way. The mean medial thickness of the pulmonary trunk was expressed as a ratio of that of the aorta. This is referred to hereafter as the PT/A ratio.

A block was taken from both lungs and sections of $5 \mu \mathrm{m}$ thickness were stained by the elastic-van Gieson technique described above. In each section the medial thickness of 10 'muscular pulmonary arteries' was measured and expressed as a percentage of the external diameter of the vessel. A mean percentage medial thickness was calculated.

At necropsy the carotid bifurcations were dissected out and fixed in $4 \%$ formaldehyde. After fixation the carotid bifurcations were processed and embedded separately in paraffin wax by a conventional technique. Serial sections, $5 \mu \mathrm{m}$ thick, were cut at intervals of $20 \mu \mathrm{m}$ and stained with haematoxylin and eosin to demonstrate the general morphology of the tissue. We used a quantitative assessment of the volumes of the carotid bodies in both groups of animals by employing Simpson's rule as quoted by Dunnill (1968). By this rule the volume, $V$, of an organ is given by

$V=1 / 3 h\left[\left(A_{0}+A_{n}\right)+4\left(A_{1}+A_{3}+\ldots A_{n}-{ }_{1}\right)+2\left(A_{2}+A_{4}+\right.\right.$ $\left.\left.\ldots A_{n}-2_{2}\right),\right]$ where $n=$ the number of sections ; $A_{0}, A_{1}$, $A_{2} \ldots=$ the area of successive sections, and $h=$ the interval between each section, which in this study was $20 \mu \mathrm{m}$.

The areas $A_{0}, A_{1}, A_{2}$ and so on were obtained by projecting each section on a sheet of graph paper with a Leitz Prado projector with a microscope head. The outline of each image was traced on the graph paper and the area enclosed by the trace was cut out with scissors. In each instance the area was calculated from the weight of the area of graph paper that had been occupied by the projected image. Calibration was carried out by weighing the area of graph paper occupied by an image of a 1-mm square projected at the same magnification. It was not necessary to weigh each area of graph paper separately because the values of the expressions $\left(A_{0}+A_{n}\right),\left(A_{1}+A_{3}+\ldots A_{n-1}\right)$ and $\left(A_{2}+A_{4}+\ldots\right.$ $\left.A_{n-2}\right)$ could be derived by weighing the relevant sheets together. No allowance was made for shrinkage of the carotid-body tissue during fixation and processing, as it was assumed that the shrinkage factor would be equal in the tissues from the three groups.

\section{RESULTS}

The initial and final total body weights in grammes are shown in Table $I$. The control

T A B L E I

INITIAL AND FINAL TOTAL BODY WEIGHTS (grammes)

\begin{tabular}{l|cl|cl}
\hline \multirow{2}{*}{ Group } & \multicolumn{2}{|c|}{ Initial } & \multicolumn{2}{c}{ Final } \\
\cline { 2 - 5 } & Range & Mean & Range & Mean \\
\hline Control & $325-425$ & 377 & $370-460$ & 415 \\
Hypoxic & $350-420$ & $387 \cdot 5$ & $250-340$ & 315 \\
Recovery & $315-420$ & $361 \cdot 5$ & $355-440$ & $393 \cdot 5$ \\
\hline
\end{tabular}

animals gained weight normally, the mean weight gain being $38 \mathrm{~g}$ during the period of the experiment. The hypoxic animals showed the characteristic loss of weight experienced by man at high altitude; in this group the mean weight loss was $72.5 \mathrm{~g}$. The weight loss was an expression of anorexia, the food consumption of the hypoxic group being lower than that of the controls. The animals allowed to recover in room air for five weeks showed a recovery of body weight, the mean weight gain in the group being $32 \mathrm{~g}$, a figure approaching that of the control group.

The values of $L V / R V$ in the three groups are shown in Table II. There was a pronounced fall of the mean ratio of the hypoxic rats from a value of 4.5 to 1.8 , indicating the development of

T A B LE I I

VALUES OF LV

$\overline{\mathbf{R V}}$

\begin{tabular}{c|c|c}
\hline Group & Range & Mean \\
\hline Control & $3 \cdot 7-5 \cdot 0$ & $4 \cdot 5$ \\
Hypoxic & $1 \cdot 2-2 \cdot 4$ & $1 \cdot 8$ \\
Recovery & $3 \cdot 3-4 \cdot 9$ & 3.9 \\
\hline
\end{tabular}


right ventricular hypertrophy. In the recovery group this ratio returned to a normal level of 3.9 , a figure almost as high as the mean figure for the controls.

The values of PT/A in the three groups are shown in Table III. There was a corresponding

T A B LE II I

VALUES OF $\frac{P T}{A}$

\begin{tabular}{c|c|c}
\hline Group & Range & Mean \\
\hline Control & $0.27-0.63$ & 0.45 \\
Hypoxic & $0.69-1.18$ & 0.95 \\
Recovery & $0.27-0.65$ & 0.51 \\
\hline
\end{tabular}

rise in the value of this ratio from 0.45 to 0.95 , indicating pronounced medial hypertrophy in the pulmonary trunk. In the recovery group this ratio returned to a normal level of $0 \cdot 51$, a figure almost as low as the mean figure for the controls.

There were pronounced histological changes in the pulmonary trunks which had undergone hypertrophy in response to chronic hypoxia, and also in those in the phase of recovery from the effects of hypoxia. The media of the pulmonary trunk of the normal Wistar albino rat is thin and largely composed of about 12 uniform, parallel elastic laminae with small amounts of interposed smooth muscle (Fig. 2). It contrasts with the aortic media which is of considerably greater thickness and which is composed of much

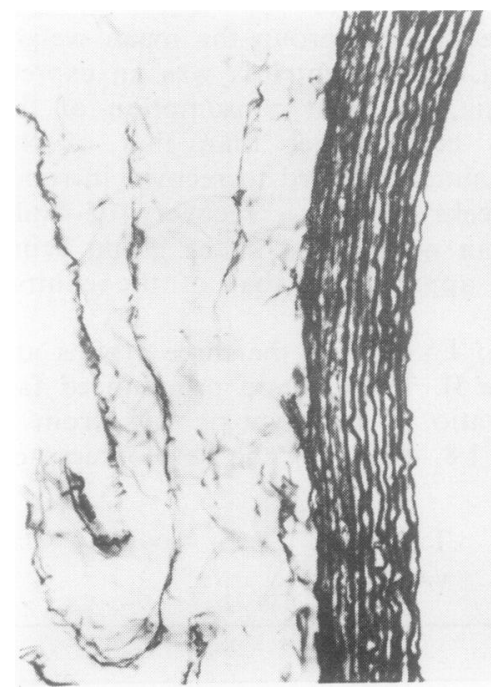

FIG. 2. Transverse section of pulmonary trunk of control rat $($ Elastic van Gieson $\times 300)$.

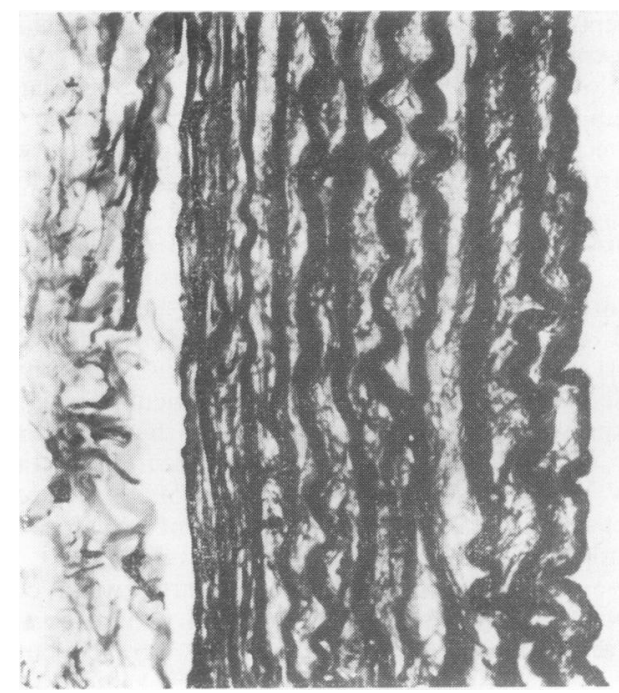

FIG. 3. Transverse section of aorta of control rat (Elastic van Gieson × 300).

thicker elastic fibres (Fig. 3). In the pulmonarye trunks of the rats which had been exposed ton chronic hypoxia without relief there was proำ nounced thickening of the media due to hyper plasia of the smooth muscle fibres situated between the elastic laminae. These had forced the elastic laminae apart and their hyperplasid in the outer part of the media had blurred the demarcation between media and adventitia (Fig $\overrightarrow{0}$ 4). The appearances were virtually identical withw

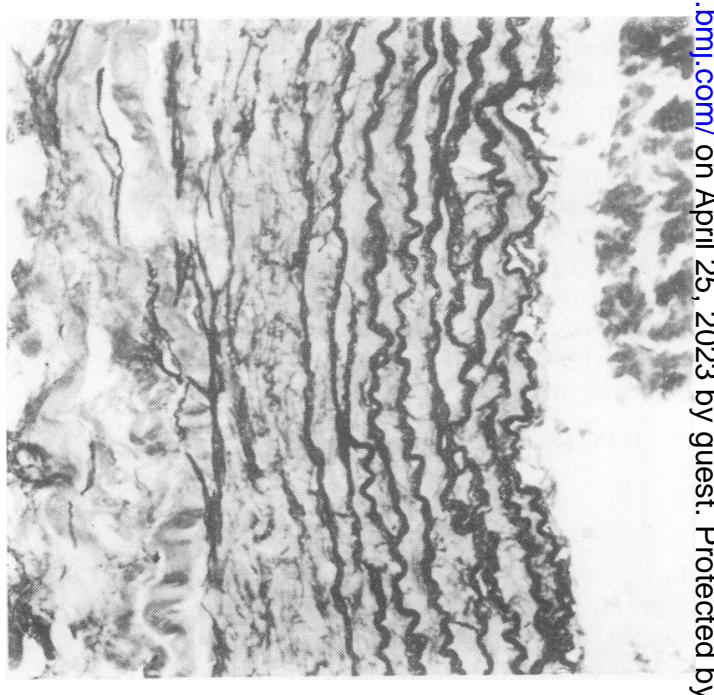

FIG. 4. Transverse section of pulmonary trunk of rat $i$ the hypoxic group (Elastic van Gieson $\times 300$ ). 
those seen in hypertrophy of the pulmonary trunk in rats in which pulmonary hypertension has been induced by the administration of Crotalaria spectabilis seeds (Heath and Kay, 1967). In the rats which had been allowed to recover from the period of chronic hypoxia in room air for five weeks the histological appearances were mid-way between those of the hypoxic and control groups. The media of the pulmonary trunk was thicker than normal (Fig. 5) but not so thick as in the

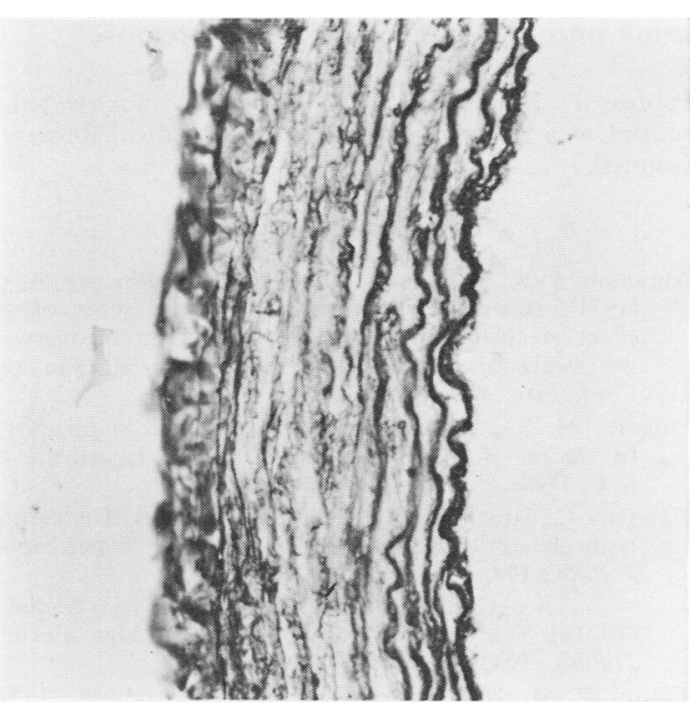

FIG. 5. Transverse section of pulmonary trunk of rat in the recovery group (Elastic van Gieson $\times 300)$.

hypoxic animals. The histological appearances of the media were still abnormal. There was an increase in inter-laminar distance, and elastic laminae were sparse in the outer media where the remains of smooth muscle hyperplasia still blurred the demarcation between media and adventitia (Fig. 5).

The volumes of the carotid body in the three groups are shown in Table IV. The mean volume of the carotid body in the hypoxic rats rose to $47.81 \mu \mathrm{m}^{3} \times 10^{6}$ compared to a figure of 13.45 $\mu \mathrm{m}^{3} \times 10^{6}$ for the controls. In the recovery group

T A B LE IV

CAROTID BODY VOLUMES $\left(\mu \mathrm{m}^{3} \times 10^{\circ}\right)$

\begin{tabular}{l|c|c}
\hline Group & Range & Mean \\
\hline Control & $6 \cdot 92-22 \cdot 23$ & 13.45 \\
Hypoxic & $19 \cdot 11-95 \cdot 03$ & 47.81 \\
Recovery & $13 \cdot 08-40.48$ & 19.82 \\
& & \\
\hline
\end{tabular}

the carotid body volume returned to a volume of $19.82 \mu \mathrm{m}^{3} \times 10^{6}$.

The values of the mean percentage medial thickness of the muscular pulmonary arteries in the three groups are shown in Table V. In none of the animals was there any suggestion of medial hypertrophy of the muscular pulmonary arteries. Statistical analysis showed no significant difference between medial thickness of muscular pulmonary arteries in the hypoxic and control groups $(t=$ $2.3138 ; \quad P<0.05)$. There was a significant difference between the medial thickness of these vessels in the hypoxic and recovery groups $(t=$ $9.0884 ; \mathrm{P}<0.001$ ), although, as stated above, there was no medial hypertrophy in either group.

\section{T A B LE V}

PERCENTAGE MEDIAL THICKNESS OF 'MUSCULAR PULMONARY ARTERIES'

\begin{tabular}{c|c|c}
\hline Group & Range & Mean \\
\hline Control & $0 \cdot 9-8 \cdot 9$ & $3 \cdot 4$ \\
Hypoxic & $0 \cdot 5-8 \cdot 2$ & $4 \cdot 3$ \\
Recovery & $0 \cdot 7-4 \cdot 9$ & $2 \cdot 5$ \\
\hline
\end{tabular}

\section{DISCUSSION}

The results of this study show that in rats exposed to a hypoxic environment simulating an altitude of $5,500 \mathrm{~m}$ above sea level there is simultaneous development of right ventricular hypertrophy, medial hypertrophy of the pulmonary trunk, and enlargement of the carotid bodies. These three anatomical changes occur rapidly and in rats are well established at the end of five weeks. After recovery from the hypoxic environment in room air for five weeks the right ventricle, pulmonary trunk, and carotid bodies return virtually to normal. The development and regression of right ventricular hypertrophy in rats under similar conditions have been reported before by Abraham, Kay, Cole, and Pincock (1971). They did not measure the medial thickness of the pulmonary trunk, which is a morbid anatomical indicator of the mean level of pulmonary arterial pressure. However, they carried out haemodynamic studies by direct cannulation of the heart and found that in hypoxia there was a progressive rise in the right ventricular mean pressure. Taken together, their haemodynamic studies and our investigation of the pathology of the pulmonary trunk confirm that in states of chronic hypoxia there is a development of pulmonary arterial hypertension which leads to the right ventricular hypertrophy already referred to. 
The basis for this increased pulmonary vascular resistance appears to be constriction of the terminal portions of the pulmonary arterial tree with the subsequent development of hypoxic hypertensive pulmonary vascular disease if the hypoxia is sustained. Abraham et al. (1971) were able to induce muscularization of the small pulmonary arterial vessels in their study. Interestingly enough, however, we were unable to induce the characteristic changes of hypoxic hypertensive pulmonary vascular disease in our animals, although the severity and duration of the hypoxia was almost identical. We do not understand the reason for this lack of reactivity of the small pulmonary arteries to hypoxia in this series, and we intend to investigate this matter further. Age and sex may be of importance in this respect for whereas the animals used by Abraham et al. were female weanling rats those we employed were adult males.

The results of our investigation appear to be applicable to human disease. They show that in hypoxic cor pulmonale, right ventricular hypertrophy and medial hypertrophy in the pulmonary trunk occur rapidly in response to chronic hypoxia. The results also show that these morbid anatomical changes are rapidly and almost totally reversible when the hypoxic stimulus is removed. This finding is in keeping with the predominantly muscular features of hypoxic hypertensive pulmonary vascular disease in man (Heath et al., 1968). The reversibility is in striking contrast to the fixed organic basis for increased, irreversible pulmonary vascular disease and resistance which is found in the late stages of, for example, large pre- and post-tricuspid congenital cardiac shunts (Heath et al., 1958). In hypoxic hypertensive pulmonary vascular disease there is very little occlusive intimal fibrosis to maintain a high fixed vascular resistance.

The other feature of the pathology which was not investigated by Abraham et al. (1971) is the enlargement of the carotid bodies occurring under hypoxic conditions with simultaneous right ventricular hypertrophy and medial hypertrophy of the pulmonary trunk. It seems likely that enlargment of the carotid bodies occurs as a diregt response of their chemoreceptor tissue to chronite hypoxia. We have noted similar enlargement of the carotid bodies before in patients with chrorfoc respiratory disease (Heath et al., 1970 ; Edwards et al., 1971a) and in animals living at high altitude (Edwards et al., 1971b). It is of considerabde interest to see that such enlargement is als rapidly reversible like the hypertrophy of the right ventricle and the media of the pulmonaity trunk once the hypoxic stimulus is removed.

Professor Heath and Dr. Edwards acknowledge receipt of a research grant from the Medical Researeh Council.

\section{REFERENCES}

Abraham, A. S., Kay, J. M., Cole, R. B., and Pincock, A. (1971). Haemodynamic and pathological study of the effect of chronic hypoxia and subsequent recovery off the heart and pulmonary vasculature of the rat. Cardiovasc. Res., 5, 95.

Dunnill, M. S. (1968). Quantitative methods in histology. In Recent Advances in Clinical Pathology, edited Fy S. C. Dyke, p. 401. Churchill, London.

Edwards, C., Heath, D., and Harris, P. (1971a). The carofid body in emphysema and left ventricular hypertrophy. J. Path., 104, 1.

- , Castillo, Y., Krüger, H., and Arias-Stella (1971b). The carotid body in animals at high altitude. J. Path., 104, 231.

Fulton, R. M., Hutchinson, E. C., and Jones, A. M. (1952). Ventricular weight in cardiac hypertrophy. Brit. He⿳亠丷厂巾t J., 14, 413.

Heath, D., Brewer, D., and Hicken, P. (1968). Cor Pulmonale in Emphysema: Mechanisms and Pathology, p. 94. Thomas, Springfield, Illinois.

and structure of the human carotid body. Its relationgto pulmonary disease and cardiac hypertrophy. Thorax, 25, 129.

_, Helmholz, F. H. Jr., Burchell, H. B., Du Shane, J. $\underset{\text { W., }}{ }$ and Edwards, J. E. (1958). Graded pulmonary vascưfar changes and haemodynamic findings in cases of ventricular and atrial septal defect and patent ductus arteriosus. Circulation, 18, 1155.

nony , and Kay, J. M. (1967). Medial thickness of pulmont of Crotalaria spectabilis seeds. Cardiovasc., Res., $1, \underset{\text { Jु }}{ }$ 